\title{
Melatonin Attenuates Her-2, p38 MAPK, p-AKT, and mTOR Levels in Ovarian Carcinoma of Ethanol-Preferring Rats
}

\author{
Grazielle M. Ferreira ${ }^{1}$, Marcelo Martinez², Isabel Cristina C. Camargo ${ }^{3}$, Raquel F. Domeniconi1, Francisco \\ Eduardo Martinez ${ }^{1}$, Luiz Gustavo A. Chuffa ${ }^{1}$ \\ 1. Department of Anatomy, Biosciences Institute, UNESP - Univ. Estadual Paulista, Botucatu-SP, Brazil, 18618-970. \\ 2. Department of Morphology and Pathology, UFSCar - Universidade Federal de São Carlos, São Carlos-SP, Brazil, $13565-905$. \\ 3. Department of Biological Sciences, Faculty of Sciences and Letters, UNESP - Univ. Estadual Paulista, Assis-SP, Brazil, $19806-900$.
}

$\square$ Corresponding author: Luiz Gustavo de Almeida Chuffa, Department of Anatomy, Bioscience Institute, UNESP - Univ. Estadual Paulista, Zip Code: 510; P.O Box: 18618-970, Rubião Júnior, s/n, Botucatu, SP - Brazil, Phone: +55 (14) 3880-0027, Fax: +55 (14) 3811-6361. E-mail: chuffa@ibb.unesp.br

( ) Ivyspring International Publisher. This is an open-access article distributed under the terms of the Creative Commons License (http://creativecommons.org/ licenses /by-nc-nd/3.0/). Reproduction is permitted for personal, noncommercial use, provided that the article is in whole, unmodified, and properly cited.

Received: 2014.07.26; Accepted: 2014.09.19; Published: 20I4.10.03

\begin{abstract}
Epidermal growth factor receptors 2 (Her-2) and 4 (Her-4) are closely associated with ovarian cancer $(\mathrm{OC})$ progression and metastasis, and a more complete understanding of these signaling pathways allow the development of new therapeutic strategies. Melatonin (Mel) is recognized as having several anticancer properties and has been reported to modulate Her-2 system in aggressive tumors. Here, we investigated $O C$ and the role of Mel therapy on the Her-2- and Her-4-signaling pathway related to downstream molecules in an ethanol-preferring rat model. To induce OC, the left ovary was injected directly with a single dose of $100 \mu \mathrm{g}$ 7, 12-dimethylbenz(a)anthracene (DMBA) dissolved in $10 \mu \mathrm{L}$ of sesame oil under the bursa. Right ovaries were used as sham-surgery controls. After developing OC, half of the animals received i.p. injections of Mel $(200 \mu \mathrm{g} / \mathrm{l} 00 \mathrm{~g}$ b.w./day $)$ for 60 days. While Mel therapy was unable to reduce Her-4 and phosphoinositide 3-kinase (PI3K) levels, it was able to suppress the OC-related increase in the levels of the Her-2, p38 mitogen-activated protein kinases (p38 MAPK), protein kinase B (phospho-AKT), and mammalian target of rapamycin (mTOR). In addition, Mel significantly attenuated the expression of Her-2, p38 MAPK, and p-AKT, which are involved in OC signaling during ethanol intake. Collectively, our results suggest that Mel attenuates the Her-2-signaling pathway in $\mathrm{OC}$ of ethanol-preferring rats, providing an effective contribution for further development of adjuvant therapies.
\end{abstract}

Key words: Ovarian cancer, melatonin, Her-2, p38 MAPK, p-AKT, mTOR

\section{Introduction}

Ovarian cancer $(\mathrm{OC})$ is one of the most lethal gynecologic cancers and has a poor prognosis when diagnosed in the late phase $(<50 \%$ with a survival rate at 5 years) [1]. Unfortunately, early-stage OC exhibit no apparent symptoms, and no effective screening tool is available [2]. OC is presumed to arise from lesions involving the ovarian surface epithelium and/or ovarian inclusion cysts [1,2], resembling morphological characteristics of the mullerian epithe- lium. Resistance to chemotherapy is a major factor limiting long-term treatment against OC [2], and paclitaxel resistance has been recognized to induce protumor activities [3,4].

The PI3K/AKT/mTOR signaling pathway has been documented in most ovarian cancers [5], and activation of this pathway is related to advanced tumor stage with poor prognosis. Studies have reported that increased expression of Her-2/neu, a member of 
the EGFR family (epidermal growth factor receptor) is directly associated to the aggressiveness and poor prognosis of breast and ovarian cancer [6,7]; however, we need better understand how these mechanisms occur in OC. Her-2 can activate several intracellular signaling pathways, leading to increased rates of proliferation, migration and survival of tumor cells [8], especially when the PI3K-AKT complex and MAPK are activated. Furthermore, tumors with increased expression of Her-2 seem to be more tolerant and resistant to endocrine therapies and chemotherapies [9]. Therefore, Her-2/neu has been the main focus for OC treatment, and its inhibition has become an important therapeutic target in clinically Her-2-overexpressing OC [10]. Conversely, Her-4, a member of the EGFR family, is weakly expressed on the ovarian surface, and it has been suggested that Her- 4 is decreased in some types of OC [11].

Because ethanol $(\mathrm{EtOH})$ intake produces a variety of co-carcinogenic effects and seems to modulate EGFR/PI3K/AKT/MAPK signaling in a dose- and time-dependent manner $[12,13]$, the potent combination of induced $\mathrm{OC}$ and $\mathrm{EtOH}$ intake are of great importance for studying appropriate histological and molecular patterns that are needed to evaluate new chemo protective compounds. Importantly, we have previously developed a useful ethanol-preferring rat model that closely reflects human OC, and, notably, long-term Mel therapy significantly reduced OC masses and the incidence of adenocarcinomas in these animals [14].

Mel (N-acetyl-5-methoxytryptamine) is an indoleamine produced by the pineal gland at night [15]. Mel exert antioxidant, immunomodulatory, and oncostatic functions [16], which inhibit the growth of tumor masses. In pharmacological concentrations, Mel showed a strong activity in inhibiting the phosphorylation of PI3K and AKT, thus deregulating this signaling pathway in breast cancer cells [17]. Interestingly, pre-treatment with selective PI3K/AKT inhibitors caused a Mel-mediated inhibitory effect on cell proliferation. In H4IIE hepatoma cells, Mel attenuated the phosphorylation of AKT and mTOR induced by hydroperoxide $\left(\mathrm{H}_{2} \mathrm{O}_{2}\right)$ radical [18]. This prevention caused by $\mathrm{H}_{2} \mathrm{O}_{2}$ on MAPK and mTOR signaling appears to be mediated via inhibition of $R a s$ protein. To date, no study has been proposed to evaluate the effects of Mel therapy upon EGFR signaling pathway in OC.

We therefore aimed to investigate the induced OC and the role of long-term Mel therapy on Her-2and Her-4-signaling pathway and related downstream molecules (p38MAPK-PI3K-p-AKT-mTOR) in an ethanol-preferring rat model.

\section{Materials and Methods}

\section{Animals and experimental design}

Eighty adult UChB (a model of ethanol-preferring rats that was developed by selective inbreeding) rats, 90-days-old, weighing $230 \mathrm{~g}$, were obtained from the Department of Anatomy, Bioscience Institute/Campus of Botucatu, UNESP - Univ Estadual Paulista. The rats were individually housed in polypropylene cages containing laboratory-grade pine shavings as bedding and maintained under constant room temperature $\left(23 \pm 1^{\circ} \mathrm{C}\right)$ and lighting conditions (12-h light/dark cycle, with the lights switched on at 6 a.m.). Filtered tap water and standard rodent chow (3074 SIF, Purina Ltda., Campinas, SP, Brazil) were provided ad libitum. All animals were divided into two arms ( $n=40$ /group): EtOH group, in which the rats had access to a $10 \%(\mathrm{v} / \mathrm{v})$ ethanol solution ad libitum (free choice of water or ethanol), and a control group, which was composed of ethanol-naïve rats without access to ethanol. When the ethanol-preferring rats reached 65 days of age, they were given a choice between two bottles containing either water or a $10 \%(\mathrm{v} / \mathrm{v})$ ethanol solution ad libitum over a period of 15 days. The animals displaying $\mathrm{EtOH}$ consumption higher than $2.0 \mathrm{~g}$ of $\mathrm{EtOH} / \mathrm{kg} /$ day (ranging from 4 to $5 \mathrm{~g}$ of $\mathrm{EtOH} / \mathrm{kg} /$ day) were selected according to the procedure outlined by Chuffa et al. $[19,20]$.

After OC development (260-days-old), the animals $(n=40)$ designated to receive Mel $(M-5250$, Sigma-Aldrich, St. Louis, MO, USA) were administered i.p. doses of $200 \mu \mathrm{g} / 100 \mathrm{~g}$ b.w. dissolved in 0.04 $\mathrm{mL}$ of $95 \% \mathrm{EtOH}$ and then diluted in $0.3 \mathrm{~mL}$ of $0.9 \%$ $\mathrm{NaCl}$ (vehicle) at a steady-state concentration of 0.3 $\mathrm{mg} / \mathrm{mL}$. The daily injections were administered nocturnally (between 18:30 - 19:00 h) over the period of 60 consecutive days [21].

Lastly, the rats were divided into four groups (n = 20): OC, composed of DMBA-induced animals that did not consume EtOH; OC+EtOH, composed of DMBA-induced animals that consumed $10 \%(\mathrm{v} / \mathrm{v})$ $\mathrm{EtOH}$ during ovarian tumor development (OTD); OC+Mel, composed of DMBA-induced animals that received Mel as therapy; and $\mathrm{OC}+\mathrm{EtOH}+\mathrm{Mel}$, composed of DMBA-induced animals that consumed 10\% (v/v) EtOH during OTD and received Mel as therapy. After the procedures, the females were anesthetized and euthanized by decapitation (during early morning at 4 a.m.) for further sample collection. The present experimental protocol was accepted by the Ethical Committee of the Institute of Bioscience/UNESP (CEEA - Permit Number: 382). 


\section{Ovarian tumor induction}

After selection for ethanol consumption, all of the animals $(\mathrm{n}=80)$ were anesthetized with $10 \%$ ketamine $(60 \mathrm{mg} / \mathrm{kg}$, i.p.) and $2 \%$ xylazine $(5 \mathrm{mg} / \mathrm{kg}$, i.p.) during the estrous phase, and a 2-cm incision was made through the skin and abdominal muscles, and the left ovaries were accessed after grasping the fat pad near the left kidney. The left ovary was injected directly under the mesovarium with a single dose of $100 \mu \mathrm{g}$ DMBA (Sigma Chemical Co, St Louis, MO) dissolved in $10 \mu \mathrm{L}$ sesame oil, which was used as the vehicle [22] and returned intact to the body cavity. The muscle layer and skin was closed using a 3-0 silk suture (Ethicon Inc., México, MX). Sham-surgery was conducted on the right ovary using only the vehicle. Antibiotic ( $10^{5}$ units of benzylpenicillin potassium) was administered i.p. for the prophylactic treatment. Over the next 180 days, tumor development was observed by ultrasonography (size and volume).

\section{Plasma Mel levels}

After blood collection, Mel was extracted from the plasma $(\mathrm{n}=20$ samples/group) using HPLC-grade methanol and separated on columns (Sep-Pak Vac C-18, reverse phase, $12.5 \mathrm{~nm}$; Water Corporation, Milford, Massachusetts, USA). Thereafter, $50 \mu \mathrm{L}$ of reconstituted samples were assayed using a Coat-a-count Melatonin ELISA Kit and immediately read at $405 \mathrm{~nm}$. The intra-assay coefficient of variation was $4 \%$, and the samples were assayed at the same time in duplicate. All reagents and microtiter plates were provided by IBL (IBL International, Hamburg, Germany), and the concentrations were reported as $\mathrm{pg} / \mathrm{mL}$.

\section{Immunohistochemistry}

Sections of OC ( $n=10 /$ group) were deparaffinized in xylene based on the areas previously identified during the morphological characterization. Tissue sections were microwaved $(700-800 \mathrm{~W})$ while immersed in $0.01 \mathrm{M}$ sodium citrate buffer, $\mathrm{pH}$ 6.0, for antigen retrieval. After blocking endogenous peroxidase activity, the tissues were incubated with 3\% BSA for $1 \mathrm{~h}$ to avoid non-specific binding. Subsequently, OC sections were incubated in a humid chamber overnight at $4^{\circ} \mathrm{C}$ with primary antibodies (Abcam, Cambridge, UK): rabbit polyclonal anti-Her-2 (1:100), rabbit monoclonal anti-Her-4 (1:100), rabbit polyclonal anti-p38MAPK (1:50), rabbit polyclonal anti-PI3K (1:100), rabbit monoclonal anti-mTOR (1:250), rabbit monoclonal anti-phospho-AKT (1:200). After immunoreactions, the slides were washed in TBS-T buffer and incubated with secondary antibody (Polymer Anti-Mouse IgG or Anti-Rabbit - DAKO ${ }^{\circledR}$ CYT) at room temperature for $1 \mathrm{~h}$. Then, the slides were re- acted with chromogen diaminobenzidine (DAB; Sigma, St. Louis, MO, USA) for 5 min. Lastly, sections were counterstained with hematoxylin. Negative controls were made by omitting the primary antibody. IHC results were analyzed under a Zeiss Axiophot II microscope (Carl Zeiss, Oberkochen, Germany) based on the levels of staining intensity as absent, weak, moderate, and strong reactions.

\section{Immunofluorescence assays}

OC cells were washed with phosphate-buffered saline (PBS, sodium chloride, potassium chloride, dihydrogen phosphate, and disodium hydrogen phosphate), fixed in $4 \%$ paraformaldehyde for $10 \mathrm{~min}$, and permeabilized with PBS at room temperature. Nonspecific binding sites were blocked with $1 \%$ bovine serum albumin (BSA, 1\%) for $60 \mathrm{~min}$. Samples were incubated with anti-Her-2 primary rabbit polyclonal antibody (dilution 1:100, overnight at $4^{\circ} \mathrm{C}$ ) followed by secondary polyclonal anti-rabbit IgG conjugated to FITC (1:200, sc-2012, Santa Cruz Biotechnology Inc., CA, USA) for $1 \mathrm{~h}$ at room temperature. Nuclei were stained with 6-diamidino-2-phenylindole (DAPI, $5 \mathrm{~min}$ ) at room temperature. Primary and secondary antibodies were diluted in blocking buffer (1\% BSA). For negative immunolabeling, no primary antibody was added. Immunopositive cells were analyzed using a fluorescence microscope (Zeiss Axiophot II, Oberkochen, Germany) at 40X magnification (excitation $590 \mathrm{~nm}$; emission filter $650 \mathrm{~nm}$ ) and for DAPI staining (excitation $365 \mathrm{~nm}$; emission filter $435-485 \mathrm{~nm}$ ). The quantification of fluorescence in merged images was performed using the Image J.

\section{Immunoblots}

OC samples were rapidly removed, and $100 \mathrm{mg}$ tissues were frozen in liquid nitrogen and stored at $80^{\circ} \mathrm{C}$. The tissues were microdissected (only areas containing serous papillary tumors were obtained) and homogenized with 10X RIPA lysis buffer (Pierce Biotechnology, Rockford, IL, USA) supplemented with a cocktail of protease inhibitors. Aliquots of 1:10 $(\mathrm{v} / \mathrm{v})$ dilutions of Triton X-100 were added to the homogenates, and samples were placed on dry ice for $2 \mathrm{~h}$ to improve extraction. Lysates were centrifuged at $21,912 \mathrm{~g}$ for $20 \mathrm{~min}$ at $4^{\circ} \mathrm{C}$ to remove insoluble material, and total protein was measured through colorimetric determination. All proteins were dissolved in 1.5 X Laemmli buffer and used for SDS-PAGE (Bio-Rad Laboratories, Hercules, CA, USA). The same amount of protein $(70 \mu \mathrm{g})$ was loaded per well and resolved into preformed $4-12 \%$ acrylamide gradient gels (Amersham Biosciences, Uppsala, Sweden) using a Tris-glycine running buffer electrophoresis system (60 mA, fixed for $2 \mathrm{~h}$ ). After electrophoresis, proteins 
were electro-transferred ( $200 \mathrm{~mA}$, fixed for $1.5 \mathrm{~h}$ ) onto nitrocellulose membranes in a Tris-glycine-methanol buffer. Prestained standards (Bio-Rad, Hercules, CA, USA) were used as molecular weight markers. Thereafter, the membranes were blocked with TBS-T solution containing $3 \%$ BSA at room temperature (RT) for $60 \mathrm{~min}$ and incubated at $4^{\circ} \mathrm{C}$ overnight with the following antibodies (1:500 in 1\% BSA): Her-2, Her-4, p38MAPK, PI3K, phospho-AKT, and mTOR (Abcam, Cambridge, UK). This treatment was followed by sequential washings in TBS-T solution and incubation for $2 \mathrm{~h}$ at RT with rabbit HRP-conjugated secondary antibodies (diluted 1:1000 in 1\% BSA). After washing in TBS-T, signals were developed using an ECL detection kit (Thermo Scientific). Immunoreactive bands were obtained from individual blots of 10 rats/group using image analysis software (NIS-Elements, Advanced Research, Nikon). $\beta$-actin were used as endogenous positive controls, and the results were expressed as the mean \pm SD. Immunoblotting concen-

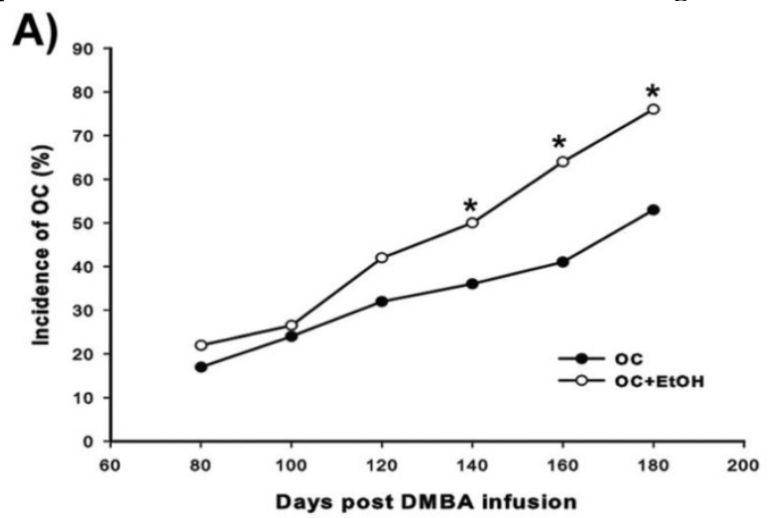

trations (\%) were represented as optical densitometry values (band intensity-pixels).

\section{Statistical analysis}

The values are presented as the mean $\pm S D$, and data analyses were performed using two-way ANOVA for two independent factors $(\mathrm{EtOH}$ consumption and Mel therapy). Significant results were subjected to post-hoc testing using Tukey's test, and statistical significance was set at $\mathrm{P}<0.05$. Sigma Plot Version 12.5 graphing software was used.

\section{Results}

The combination of EtOH intake with DMBA promoted a high incidence of OC after 140 days of age (Fig. 1A); all differential stages of OC (from 4 to 25 weeks after induction) were recorded during the development (Fig. 1B), and no evidence of altered macroscopic phenotype was observed following $\mathrm{EtOH}$ consumption.

\section{B)}
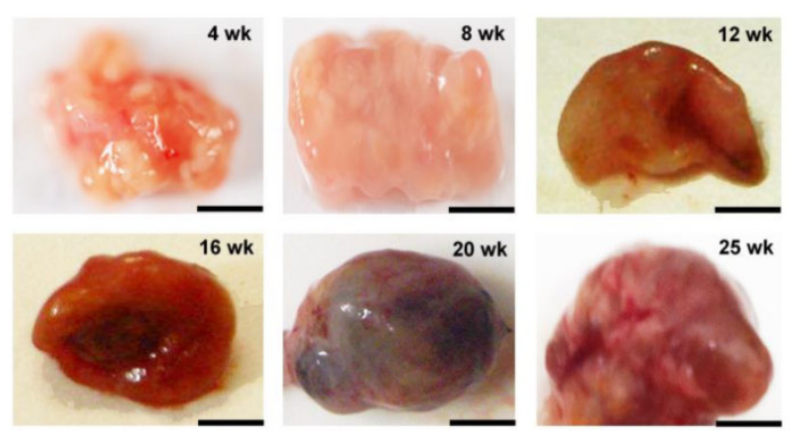

Figure I. Development of OC. (A) Cumulative incidence of OC from 80 to 180 days post-DMBA injection. The combination of EtOH with DMBA showed a significant incidence (\%) of tumor development from I40 to I 80 days post OC induction. (B) Anatomopathological specimens of OC from 4 week (wk) until 25 wk after tumor induction. Bar $=5 \mathrm{~mm},{ }^{*} \mathrm{P}<0.05$.

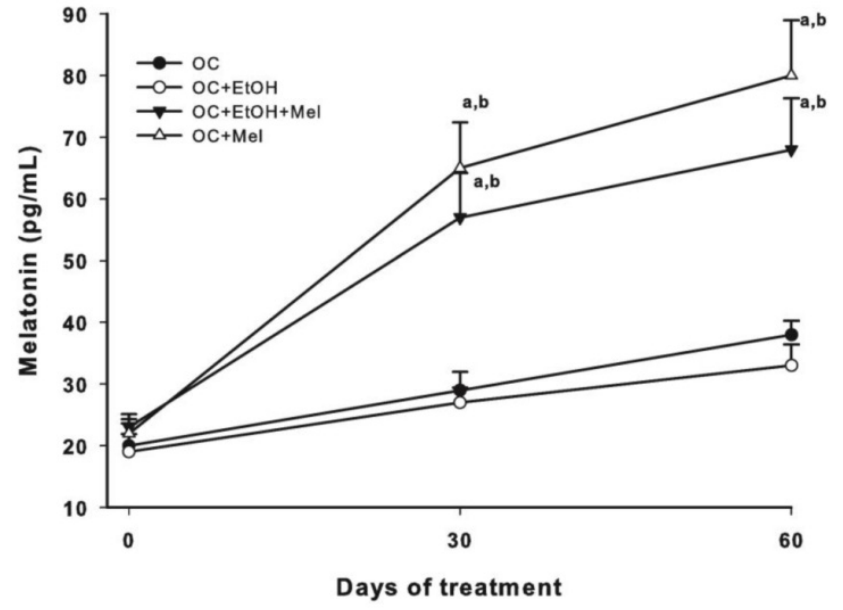

Figure 2. Plasma melatonin levels $(\mathrm{pg} / \mathrm{mL})$. Concentration of Mel prior to treatment initiation (day 0 ) and at 30 and 60 days of treatment in all experimental groups ( $\mathrm{n}=10 /$ group). a $\mathrm{P}<0.05$ versus Group $\mathrm{OC}$ and b $\mathrm{P}<0.05$ versus Group $\mathrm{OC}+\mathrm{EtOH}$.
To validate the treatment, plasma Mel levels were measured at 30- and 60-day treatment, and efficiently, OC+Mel and OC+EtOH+Mel groups showed increased levels of circulating Mel (Fig. 2). In response to Mel therapy, the expression and immunostaining of Her-2 was decreased in the OC+Mel group (1.59-fold reduced vs. OC group) and in the $\mathrm{OC}+\mathrm{EtOH}+\mathrm{Mel}$ group (1.33-fold reduced vs. OC+EtOH group) in serous papillary OC (Fig. 3 A-D, I, J). In contrast, the expression of Her-4 was unchanged along the treatments (Fig. 3 E-H, I, K). Immunofluorescence assays detected the level and localization of Her-2 in OC cells. Mel therapy resulted in downregulation of Her-2 expression (Fig. 3 L, M; fluorescence level reduced from $88 \% \pm 9.2(\mathrm{OC})$ to $46 \% \pm 11.6(\mathrm{OC}+\mathrm{Mel}))$. Interestingly, $\mathrm{EtOH}$ intake significantly increased Her-2 levels in OC, and Mel therapy drastically decreased these levels (Fig. $3 \mathrm{~N}$, O; fluorescence level reduced from $113 \% \pm 13.2(\mathrm{OC}+\mathrm{EtOH})$ to $63 \% \pm 10.1$ $(\mathrm{OC}+\mathrm{EtOH}+\mathrm{Mel}))$. 

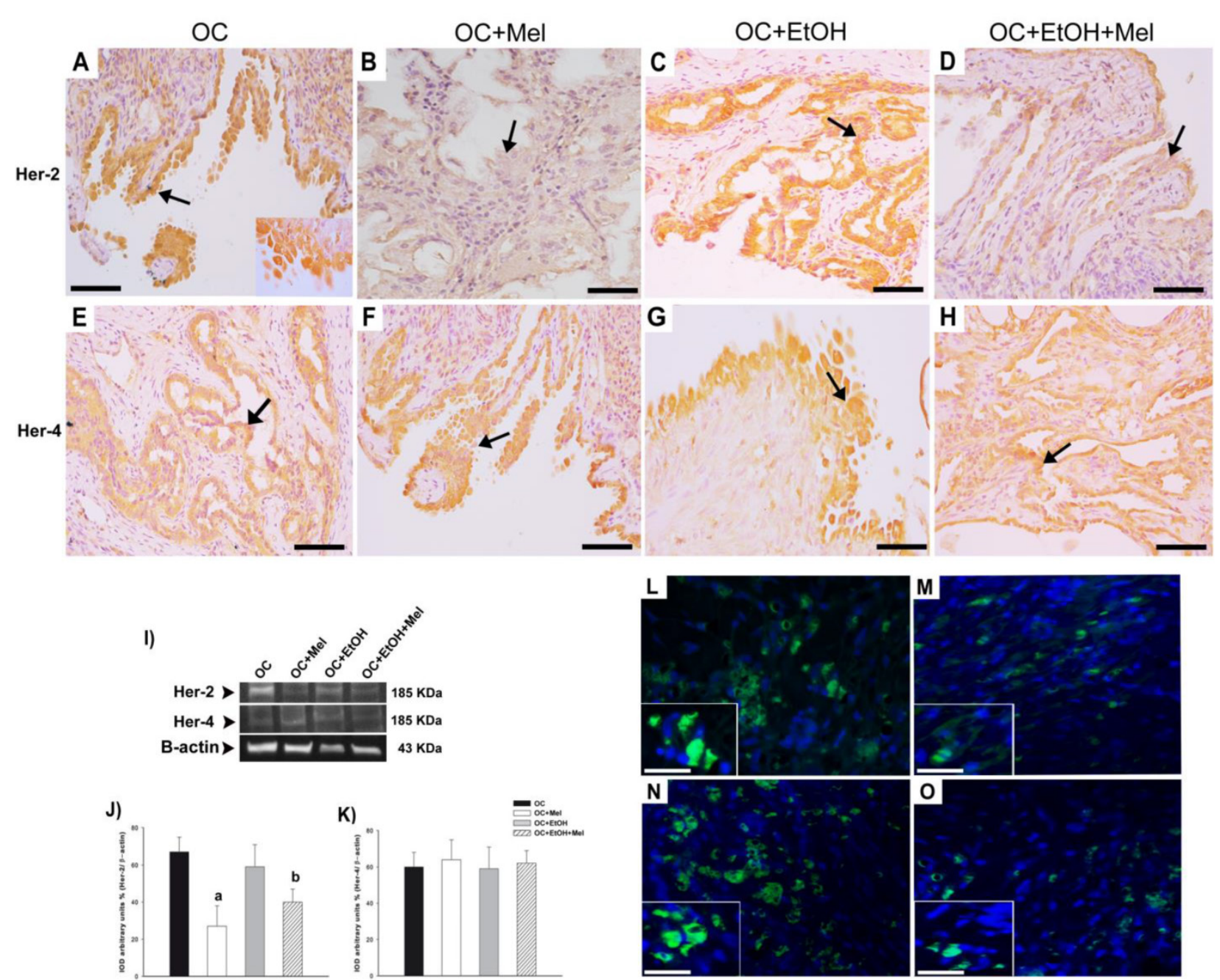

Figure 3. Immunohistochemical/fluorescence localization and Western blot analysis of Her-2 and Her-4 in serous papillary OC. The immunoreaction of Her-2 was intense in the surface epithelium of the $\mathrm{OC}(\mathrm{A})$ and $\mathrm{OC}+\mathrm{EtOH}(\mathrm{C})$ groups (arrows), but not in the stroma. Absence of reaction to Her- 2 was observed in the OC+Mel $(\mathrm{B})$ group (arrow), while a weak Her-2 reaction appeared in the epithelium of the OC+EtOH+Mel (D) group (arrow). A moderate Her-4 reaction was similar in the epithelium of the OC (E), OC+Mel (F), OC+EtOH (G), and OC+EtOH+Mel (H) groups (arrow). Bar $=20 \mu \mathrm{m}$. Negative controls were used. (I) Representative Her-2 and Her-4 profiles of extracts (70 $\mu \mathrm{g}$ protein) pooled from 10 samples/group (upper panel). (J-K) Extracts obtained from individual animals were used for densitometric analysis of the Her-2 and Her-4 levels following normalization to the $\beta$-actin. All results are expressed as the mean $\pm S D(n=10)$. a $P<0.05$ versus $O C$; ${ }^{b} P<0.05$ versus $O C+E t O H$. (L-O) Merged images of the immunofluorescence of Her-2 and DAPI nuclear staining in OC (L), OC+Mel (M), OC+EtOH (N) and OC+EtOH+Mel $(\mathrm{O})$; details of the cell receptor staining $(\mathrm{Alexa}$ fluor $\AA 488$, Bar $=10 \mu \mathrm{m})$

To investigate the effects of Mel upon Her-2-signaling pathway, the downstream molecules p38 MAPK, PI3K, p-AKT, and mTOR were measured in OC tissues. Curiously, Mel therapy downregulated p38 MAPK in the groups OC+Mel (1.46-fold reduced vs. OC; Fig. 4 A, B, Q, R) and $\mathrm{OC}+\mathrm{EtOH}+\mathrm{Mel}$ (1.29-fold reduced vs. OC+EtOH; Fig. 4 C, D, Q, R). While no significant differences $(\mathrm{P}>0.05)$ were found in the expression of PI3K over the treatments (Fig. 4 E-H, Q, S), Mel therapy efficiently downregulated p-AKT in the groups OC+Mel (1.45-fold reduced vs. OC; Fig. 4 I, J, Q, T) and OC+EtOH+Mel (1.24-fold reduced vs. OC+EtOH; Fig. 4 K, L, Q, T). In addition, Mel therapy reduced the expression of mTOR in the OC+Mel group (1.37-fold reduced vs. OC; Fig $4 \mathrm{M}, \mathrm{N}$, $\mathrm{Q}, \mathrm{U})$, but not in the presence of EtOH (P > 0.05; Fig 4 $\mathrm{O}, \mathrm{P}, \mathrm{Q}, \mathrm{U})$. Briefly, Figure $4 \mathrm{~V}$ indicates the positive or negative regulatory signals produced by Mel therapy or EtOH intake on Her-2 activation, and related downstream molecules (p38 MAPK, p-AKT, mTOR) in the "in vivo" OC cell.

\section{Discussion}

We have previously reported that Mel therapy efficiently reduced OC masses (size and volume) as well as the incidence of some adenocarcinomas in ethanol-preferring animals [14]. Here, our results supported a slight increase of circulating Mel during the treatment in both Mel-treated groups at night, even in the presence of EtOH. Notably, this is the first study to demonstrate that Mel therapy negatively regulated the expression of Her-2, p38 MAPK, p-AKT, and mTOR, but not Her-4 and PI3K levels in OC of ethanol preferring-rats. 

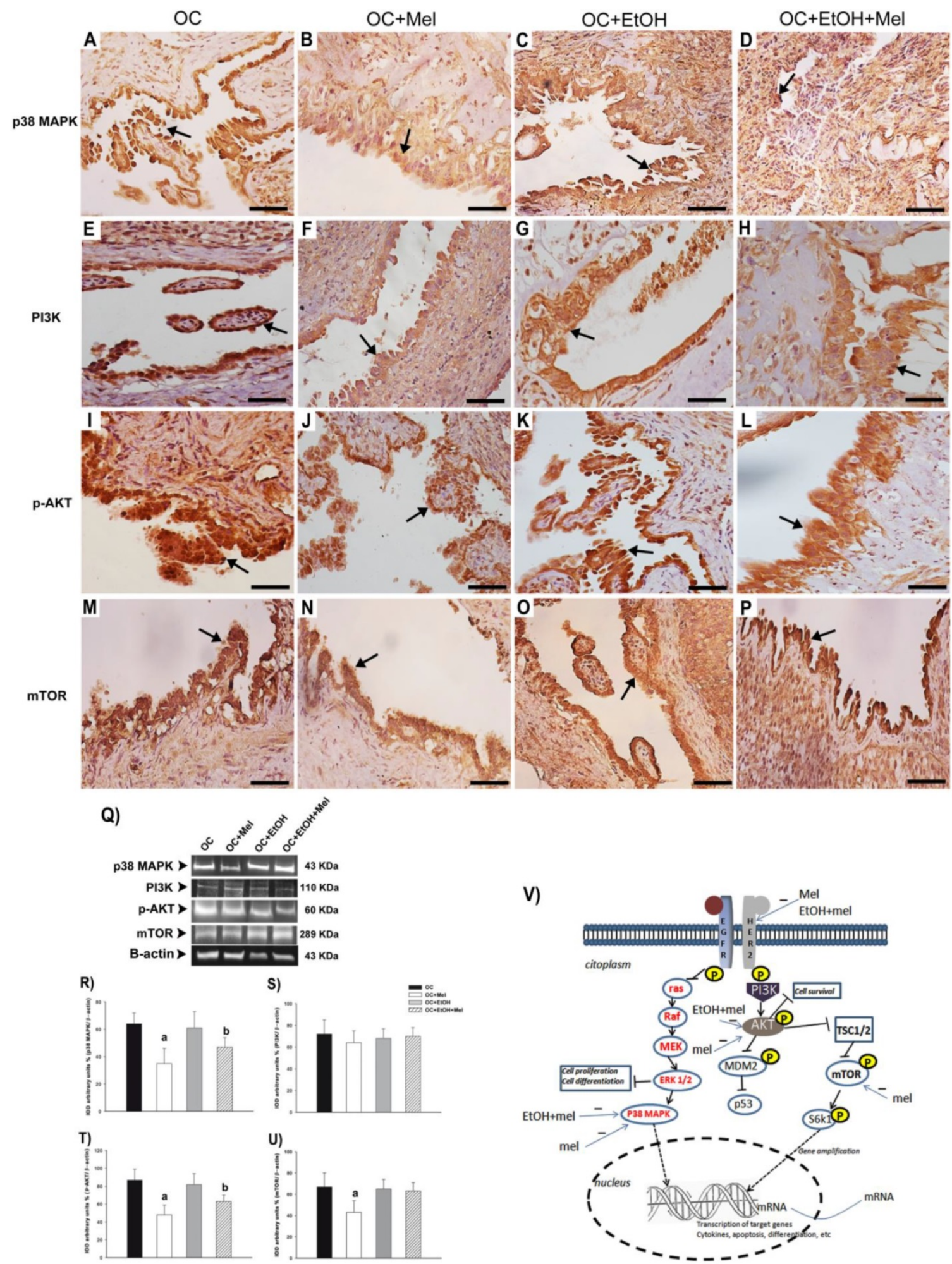

Figure 4. Immunohistochemical localization and Western blot analysis of p38 MAPK, PI3K, p-AKT, and mTOR in serous papillary OC. The immunoreaction of p38 MAPK was moderate in the OC (A) and OC+EtOH (C) groups while a weak reaction was observed in OC+Mel (B) and OC+EtOH+Mel (D) animals (arrow). Immunoreaction of PI3K was only moderate to weak in the surface epithelium of the $\mathrm{OC}(\mathrm{E})$ and a weak reaction was notable in the $\mathrm{OC}+\mathrm{Mel}(\mathrm{F}), \mathrm{OC}+\mathrm{EtOH}(\mathrm{G})$ and $\mathrm{OC}+\mathrm{EtOH}+\mathrm{Mel}$ (H) groups (arrow). A strong reaction to p-AKT was present in the papillae epithelium of the $\mathrm{OC}(\mathrm{I})$ and $\mathrm{OC}+\mathrm{EtOH}(\mathrm{K})$ groups (arrows). The $\mathrm{OC}+\mathrm{Mel}(\mathrm{l})$ and $\mathrm{OC}+\mathrm{EtOH}+\mathrm{Mel}$ (L) groups showed moderate and weak signals, respectively, for P-AKT (arrows). Reaction of mTOR was only weak in the OC+Mel (N) group, in contrast to moderate to high signals in the $\mathrm{OC}(\mathrm{M}), \mathrm{OC}+\mathrm{EtOH}(\mathrm{O})$, and $\mathrm{OC}+\mathrm{EtOH}+\mathrm{Mel}(\mathrm{P})$ grups. Bar $=20 \mu \mathrm{m}$. Negative controls were used. (Q) Representative p38 MAPK, PI3K, p-AKT, and mTOR profiles of cytosolic extracts (70 ug protein) pooled from 10 samples/group (upper panel). (R-U) Extracts obtained from individual animals were used for densitometric analysis of the proteins following normalization to house-keeping genes ( $\beta$-actin). All results are expressed as the mean $\pm S D(n=10)$. a $P<0.05$ versus $O C ; \mathrm{b} P<0.05$ versus $O C+E t O H$. (V) Schematic representation of the EGFR/Her-2 signaling pathway leading to the activation of downstream molecules in OC cell. This activation occurs after the ligand binds to its specific receptor. Intracellular signaling results in the phosphorylation of PI3K, AKT, mTOR, S6kI, and ras. Lastly, P38 MAPK and S6kI may be translocated to the nucleus to promote the transactivation of target genes related to inflammation, apoptosis, differentiation, and cell proliferation. Her-2, p38 MAPK, and p-AKT are downregulated by Mel and/or EtOH+Mel. Mel therapy also negatively regulated mTOR expression in OC. 
Dysregulated Her-2 signaling in OC results from either gene amplification or overexpression and promotes faster cell growth, improved DNA repair, and increased colony formation $[23,24]$. It is associated with an elevated risk of progression and death among women with both early and late stages of disease, and furthermore, high expression of Her-2 is assumed to cause resistance to endocrine therapies and chemotherapies [9]. Mel therapy significantly reduced the expression and immunostaining of Her-2, which would appear to make Mel an attractive molecule for targeted immunotherapies in patients with Her2-positive OC. Although Mel can mobilize antitumor immune responses [14], it also functions by disrupting Her-2 signaling in primary ovarian carcinomas [25]. Despite a lower expression of Her-4 has been observed in OC cells, no effect of Mel therapy was found along the experimental groups. In breast cancer cells (MCF-7/6 and MCF-7/Her2.1 lineages), melatonin at doses of $10^{-9} \mathrm{M}$ showed a suppressive effect on the growth of these cells [26]. According to previous studies, overexpression of Her-2/neu and activation of the Her2/neu signaling pathway increases receptor tyrosine phosphorylation, thus producing effects on cellular transformation [27], and can constitutively activate p38 MAPK [28], while promoting OC cell invasion and metastasis.

Regulation of MAPKs by Mel has been observed in several cell types, including the CRL 1999 and NCI-H292 cells, where Mel has been reported to inhibit the phosphorylation of ERK1/2 and JNK (c-Jun N-terminus kinase) [29,30]. Surprisingly, we found that Mel therapy efficiently reduced the expression of p38 MAPK, p-AKT, and mTOR in OC cells. Importantly, downregulation of the p38 MAPK as well as Her-2-related downstream molecules, such as AKT and mTOR, may prevent the OC cells to proliferate, migrate, and metastasize [31]. In this context, positive Her-2-expressing ovarian tumors may be eligible for Mel treatment. Nevertheless, whether Mel affect directly or indirectly these downstream molecules remains unclear, and may be investigated in future studies.

In this experimental model of animals bearing OC, Mel therapy significantly downregulated Her-2, p38MAPK, and p-AKT even in the presence of EtOH consumption. Indeed, $\mathrm{EtOH}$ per se is associated to the activation of intracellular signaling that lead to p38MAPK and PI3K phosphorylation [32], thus promoting a beneficial microenvironment to the OC cell. Activation of EGFR by the EtOH was inversely correlated to the expression of cell adhesion proteins, such as E-cadherins, which can promote cell migration, and further tumor progression, in a dose- and tissue-dependent manner [33]. Because no suppres- sion of EtOH intake was observed, we suggest that Mel directly affected the metabolism of EtOH, which indirectly was reflected by downregulation in Her-2-signaling pathway. Although mTOR expression seems to be unaffected by $\mathrm{EtOH}$ intake, Mel therapy significantly inhibited their expression in experimental OC. Recent evidences have reported that Mel induces downregulation of the phosphorylation of mTOR and AKT, thus attenuating the expression of survival genes, such as McL-1, Bcl-xL, cyclin D1, and cyclin E in treated MDA-MB-231 breast cancer cells [34], and in hepatoma H22 cells [35]. Also reinforcing our findings, Wang et al. [17] showed that pharmacological concentrations of Mel are able to cause inhibition of the PI3K/AKT phosphorylation in breast cancer. Moreover, previous treatment with selective inhibitors of the AKT blocked the Mel-mediated inhibition on cell proliferation. In this context, we launched first evidence indicating that Mel, when administered at pharmacological doses, may partially act through the same mechanism in OC cells.

The relationship between oxidative stress and the antioxidative properties of Mel have been well documented $[21,36]$, and high production of reactive oxygen species (ROS) is strongly associated to cell damage and cancer. Notably, Mel treatment has prevented many of the $\mathrm{H}_{2} \mathrm{O}_{2}$-induced alterations in the MAPK and mTOR signaling pathways in H4IIE hepatoma cells [18]. Despite the well known effect of Mel as ROS scavenger and by exerting antitumor activities, the exact role of Mel on Her-2-related $\mathrm{PI} 3 \mathrm{~K} / \mathrm{AKT} / \mathrm{mTOR}$ signaling pathway in the process of cancer progression still need to be further investigated.

In summary, the present study investigated the cross-talk between the Her-2- and Her- 4 system and Mel therapy in an ethanol-preferring rat model for OC. Although Mel has unaffected the Her-4, it is able to promote downregulation of the Her-2 and related downstream molecules, such as p38 MAPK, p-AKT, and $\mathrm{mTOR}$ in these animals bearing OC. Furthermore, Mel therapy was efficient in reducing Her-2, p38 MAPK, and p-AKT in OC of ethanol-drinking animals. Overall, these findings indicate that the inhibition of Her-2 signaling by Mel may critically be involved with their antitumor effect in aggressive OC, thus suggesting $\mathrm{Mel}$ as an adjuvant drug for $\mathrm{OC}$ chemotherapies.

\section{Acknowledgments}

We are grateful to Mr Thiago da Silva and Mr. Gelson Rodriguez from Department of Anatomy, IBB/UNESP, Botucatu-SP, for excellent technical support. 


\section{Financial support}

We would like to give a special thanks to FAPESP (Fundação de Amparo à Pesquisa do Estado de São Paulo, Proc. 2013/10309-9, 2013/02466-7) by providing financial support.

\section{Author contributions}

LGAC, FEM, GMF: collected and analyzed the data, drafted the manuscript, and conceived the main idea of the study. MM, ICCC, RFD: participated in the acquisition of data and in the design and intellectual conception of the study. All authors performed the statistical analysis and approved the final version of the manuscript.

\section{Competing Interests}

The authors have declared that no competing interest exists.

\section{References}

1. Cannistra SA. Cancer of the ovary. New Engl J Med. 2004; 351: 2519-65.

2. Fallows S, Price J, Atkinson RJ, et al. P53 mutation does not affect prognosis in ovarian epithelial malignancies. J Pathol. 2001; 194: 68-75.

3. Kelly MG, Alvero AB, Chen R, et al. TLR-4 signaling promotes tumor growth and paclitaxel chemoresistance in ovarian cancer. Cancer Res. 2006; 66: 3859-68.

4. Chen R, Alvero AB, Silasi DA, et al. Cancers take their Toll-the function and regulation of Toll-like receptors in cancer cells. Oncogene. 2008; 27: 225-33.

5. Sheng Q, Liu J. The therapeutic potential of targeting the EGFR family in epithelial ovarian cancer. British J Cancer. 2011; 104: 1241-5.

6. Meric-Berstam F, Hung MC. Advances in targeting human epidermal growth factor receptor-2 signaling for cancer therapy. Clin Cancer Res. 2006; 12: 6326-30

7. Reese DM, Slamon DJ. HER-2/neu signal transduction in human breast and ovarian cancer. Stem Cells. 1997; 15: 1-8.

8. Baselga J, Swain SM. Novel anticancer targets: revisiting ERBB2 and discovering ERBB3. Nat Rev Cancer. 2009; 9: 463-475.

9. Burstein HJ. The distinctive nature of HER2-positive breast cancers. N Engl J Med. 2005; 353: 1652-4.

10. Yang H-L, Lin K-Y, Juan Y-C, et al. The anti-cancer activity of Antrodia camphorata against human ovarian carcinoma (SKOV-3) cells via modulation of HER-2/neu signaling pathway. J Ethnopharmacol. 2013; 148: 254-65.

11. Srinivasan R, Poulsom R, Hurst $\mathrm{HC}$, et al. Expression of the c-erb-4/HER4 protein and mRNA in normal human fetal and adult tissues and in a survey of nine solid tumour types. J Pathol. 1998; 185: 236-45.

12. Zeng T, Zhang CL, Song FY, et al. PI3K/AKT pathway activation was involved in acute ethanol-induced fatty liver in mice. Toxicology. 2012; 296: 56-66.

13. Corl AB, Berger KH, Ophir-Shohat G, et al. Happy hour, a Ste 20 family Kinase, implicates EGFR signaling in ethanol-induced behaviors. Cell. 2009; 137: 949-60.

14. Chuffa LG, Fioruci-Fontanelli BA, Mendes LO, et al. Characterization of chemically induced ovarian carcinomas in an ethanol-preferring rat model: influence of long-term melatonin treatment. Plos One. 2013; 8: e81676.

15. Stehle JH, Saade A, Rawashdeh O, et al. A survey of molecular details in the human pineal gland in the light of phylogeny, structure, function and chronobiological diseases. J Pineal Res. 2011; 51: 17-43.

16. Reiter RJ. Mechanisms of cancer inhibition by melatonin. J Pineal Res. 2004; 3: 213-4.

17. Wang J, Xiao X, Zhang $\mathrm{Y}$, et al. Simultaneous modulation of COX-2, $\mathrm{p}$ 300, Akt, and Apaf-1 signaling by melatonin to inhibit proliferation and induce apoptosis in breast cancer cells. J Pineal Res. 2012; 53: 77-90.

18. Kimball SR, Abbas A, Jefferson LS. Melatonin represses oxidative stress-induced activation of the MAP kinase and mTOR signaling pathways in H4IIE hepatoma cells through inhibition of Ras. J Pineal Res. 2008; 44: 379-86.
19. Chuffa LG, Seiva FR, Fávaro WJ, et al. Melatonin and ethanol intake exert opposite effects on circulating estradiol and progesterone and differentially regulate sex steroid receptors in the ovaries, oviducts, and uteri of adult rats. Reprod Toxicol. 2013; 39: 40-9.

20. Chuffa LG, Seiva FR, Fávaro WJ, et al. Melatonin reduces LH, 17 beta-estradiol and induces differential regulation of sex steroid receptors in reproductive tissues during rat ovulation. Reprod Biol Endocrinol. 2011; 9: 108.

21. Chuffa LG, Amorim JP, Teixeira GR, et al. Long-term melatonin treatment reduces ovarian mass and enhances tissue antioxidant defenses during ovulation in the rat. Braz J Med Biol Res. 2011; 44: 217-23.

22. Hoyer PB, Davis JR, Bedrnicek JB, et al. Ovarian neoplasm development by 7,12-dimethylbenz[a]anthracene (DMBA) in a chemically-induced rat model of ovarian failure. Gynecol Oncol. 2009; 112: 610-5.

23. Tuefferd M, Couturier J, Penault-Llorca F, et al. Her2 status in ovarian carcinomas: A multicenter GINECO study of 320 patients. Plos One. 2007; 2: e1138.

24. Yarden $Y$, Sliwkowski MX. Untangling the ErbB signalling network. Nat rev mol cell biol. 2011; 2: 127-37.

25. Lanitis E, Dangaj D, Hagemann IS. Primary human ovarian epithelial cancer cells broadly express HER2 at immunologically-detectable levels. Plos one. 2012; 7: e49829.

26. Mao L, Yuan L, Slakey ML, et al. Inhibition of breast cancer cell invasion by melatonin is mediated through regulation of the p38 mitogen-activated protein kinase signaling pathway. Breast Cancer Research. 2010; 12: R107.

27. Chazin V, Kaleko M, Miller A, et al. Transformation mediated by the human HER-2 gene independent of the epidermal growth factor receptor. Oncogene. 1992; 7: 1859-66.

28. Oh AS, Lorant LA, Holloway JN, et al. Hyperactivation of MAPK induces loss of ERa expression in breast cancer cells. Mol Endocrinol. 2001; 15: 1344-59.

29. Shin IS, Park JW, Shin NR, et al. Melatonin inhibits MUC5AC production via suppression of MAPK signaling in human airway epithelial cells. J Pineal Res. 2014; 56: 398-407.

30. Shi D, Xiao X, Wang J, et al. Melatonin suppresses proinflammatory mediators in lipopolysaccharide-stimulated CRL1999 cells via targeting MAPK, NF-kB, c/EBP $\beta$, and p300 signaling. J Pineal Res. 2012; 53: 154-65.

31. Baselga J, Swain SM. Novel anticancer targets: revisiting ERBB2 and discovering ERBB3. Nat Rev Cancer. 2009; 9: 463-75.

32. Ke Z, Lin H, Fan Z, et al. MMP-2 mediates ethanol-induced invasion of mammary epithelial cells over-expressing ErbB2. Int J Cancer. 2006; 119: 8-16.

33. Meng Q, Gao B, Goldberg ID, et al. Stimulation of cell invasion and migration by alcohol in breast cancer cells. Biochem Biophy Res Commun. 2000; 273: 448-53.

34. Jung JH, Sohn EJ, Shin EA, et al. Melatonin suppresses the expression of $45 \mathrm{~S}$ preribosomal RNA and upstream binding factor and enhances the antitumor activity of puromycin in MDA-MB-231 breast cancer cells. Evid Based Complement Alternat Med. 2013; 2013: 879746.

35. Liu C, Jia Z, Zhang $X$, et al. Involvement of melatonin in autophagy-mediated mouse hepatoma H22 cell survival. Int Immunopharmacol. 2012; 12: 394-401.

36. Chuffa LG, Amorim JP, Teixeira GR, et al. Long-term exogenous melatonin treatment modulates overall feed efficiency and protects ovarian tissue against injuries caused by ethanol-induced oxidative stress in adult UChB rats. Alcohol Clin Exp Res. 2011; 35: 1498-508. 